\title{
CELL SEPARATION AND GENE EXPRESSION ANALYSIS IN A TUMOR-STROMA INTERACTION MODEL
}

\author{
REGINE DAHSE AND HARTWIG KoSMEHL \\ Institute of Pathology, HELIOS Clinic Erfurt, Germany \\ e-mail: rdahse@erfurt.helios-kliniken.de, hkosmehl@erfurt.helios-kliniken.de \\ (Accepted May 28, 2004)
}

\begin{abstract}
A novel technique for co-culturing and separating fibroblasts and carcinoma cells in a 2-D model of tumorstroma interaction is presented. The methodology is based on cell co-cultivation on an $1.35 \mu \mathrm{m}$ thin membrane followed by rapid immunostaining and microdissection of the different cell compartments using a laser microdissection system (P.A.L.M. Microlaser Technologies AG, Germany). For identifying the tumor cell compartment, immunolabeling for a marker that is expressed only in epithelial tumor cells is performed. The RNA quality from the microdissected co-cultured cells was successfully proved by RT-PCR for a housekeeping gene transcript and for the laminin gamma 2 chain gene transcript used before in the tumor cell immunostaining. Laminin cDNA was amplificable only in tumor cells and not in the co-cultivated fibroblasts indicating no cell-cross-contamination during microdissection. Microdissected tumor and stroma cells from the presented membrane based co-culture model can be used for gene expression profiling and DNA based analysis in the investigation of tumor-stroma interactions.
\end{abstract}

Keywords: 2-D-coculture model, gene expression, laser microdissection, tumor invasion, stroma.

\section{INTRODUCTION}

Cellular adhesion, cell migration and invasion are essential processes in tumor progression and do include actions by malignant cells as well as by the stromal microenvironment. Tumor cells invade the stroma containing fibroblasts, endothelial cells and an extracellular matrix (ECM) composed mainly of collagens and proteoglycans, and containing numerous growth factor precursors. Tumor cells express matrix metalloproteinases (MMP) and secrete growth factors, some of which may behave as chemokines. As a result of direct physical contact with stromal cells and the effect of their growth factors, tumor cells induce fibroblast activation, endothelial cell proliferation and leucocyte recruitment. Activated fibroblasts express MMP and secrete growth factors and ECM components; endothelial cells also produce growth factors and express MMP, and leucocytes display an activated phenotype, contributing their own MMP and a variety of cytokines and chemokines. The combined effect of activity derived from both the tumor and stromal cells accelerates ECM degradation and growth factor release. The net effect on the tumor cells is promotion of migration, invasion, survival and proliferation (Stamenkovic, 2003).
In vitro, tumor cell - stromal fibroblast interaction and tumor-induced alterations in the fibroblast-specific ECM production can be studied by 2-D and 3-D coculture models (Kunz-Schughart et al., 2001). In conventional coculture systems, the different cell types are grown together in glass-slide based chambers with medium. For the analysis of tumor/stroma interactioninduced modifications, histology and immunohistochemistry can be performed with standard protocols. For specific PCR, Western Blot and gene expression analysis in fibroblasts and/or tumor cells, highly pure cell populations are required. The instrumentation should be able to separate the cocultured cells into clearly different compartments. This can be done, e.g., by flow cytometry, fluorescence- activated cell sorting (FACS) or magnetic cell separation (MACS) using fibroblast-specific antibodies or anti-fibroblast microbeads, however, the process on separation will often be more laborious than the process of cocultivation itself.

Here we present an alternative technique for coculturing and separating fibroblasts and carcinoma cells. It is based on cell co-cultivation on a $1.35 \mu \mathrm{m}$ thin membrane combined with laser capture microdissection of the cell compartments after rapid immunolabeling. Laser microdissection instruments 
enable to define the region of a stained tissue slide to excise. The cells of interest are isolated from their surroundings with a clear-cut gap by laser microdissection. Laser treatment does not impair DNA, RNA or protein recovery. The selected cell cluster or even single cells are transferred directly into a collection tube.

It is shown that our technique of laser microdissecting tumor and fibroblast cells from the presented membrane based coculture model maintains the integrity of the cells and allows the extraction of intact RNA from a limited number of cells. The cultured cells can be seen by normal microscopy and the dissection of cells can be followed by microscopy, also during the microdissection procedure. The cell compartments can be separated without cell crosscontamination after immunostaining as a tool for expression profiling and genetic analysis.

\section{MATERIALS AND METHODS}

\section{MATERIALS}

A tumor cell line as a model of an invasive tumor and a human fibroblast cell line as an artificial stroma compartment is used for co-cultivation (Dahse et al., 2002a).

\section{METHODS}

Figure 1 summarises the methodology in a flow chart.

2-D coculture of tumor cells and fibroblasts: About $5 \times 10^{4}$ fibroblasts were allowed to adhere on $2 \times 2 \mathrm{~cm}$ membrane fragments floating in cell culture medium, cultured until showing a subconfluent monolayer and subsequently overlayed with $5 \times 10^{4}$ carcinoma cells as described previously (Dahse et al., 2002a). This special membrane (P.A.L.M. Microlaser Technologies AG, Germany) has been originally developed for the mounting of histological tissue sections on regular glass slides mainly to hold the microdissected area or specimen together during the catapulting procedure with the laser microdissection system. Recently, new commercial accessories for live cell handling with also for co-culture models in tumor-stroma interaction studies. These are disposable cell culture dishes $(5 \mathrm{~cm}$ in diameter) containing a double membrane layer (please see for details the application page of http://www.palmmicrolaser.com). The biological carrier membrane plus the supporting thin laser microdissection membrane enable cultivation and microdissection of adherent growing living cells. Even recultivation of the isolated cell compartments is possible in order to obtain homogenous cell populations.

Co-culture of tumor and stroma cells is performed for 6 - 10 days.

Immunohistochemistry: For identifying the tumor cell compartment, immunolabeling is performed for a marker that is expressed only in tumor cells, such as laminin. Alternatively, other established tumor cell markers such as cytokeratine can be used. Modified immunostaining has to be used in order to identify the tumor cells and maintain the DNA and RNA integrity for subsequent analysis as described in Dahse et al. (2002a). For counterstaining the stroma cells, a short protocol with hematoxylin can be used, but is not mandatory for visualisation.

Figure: Methodology flow chart

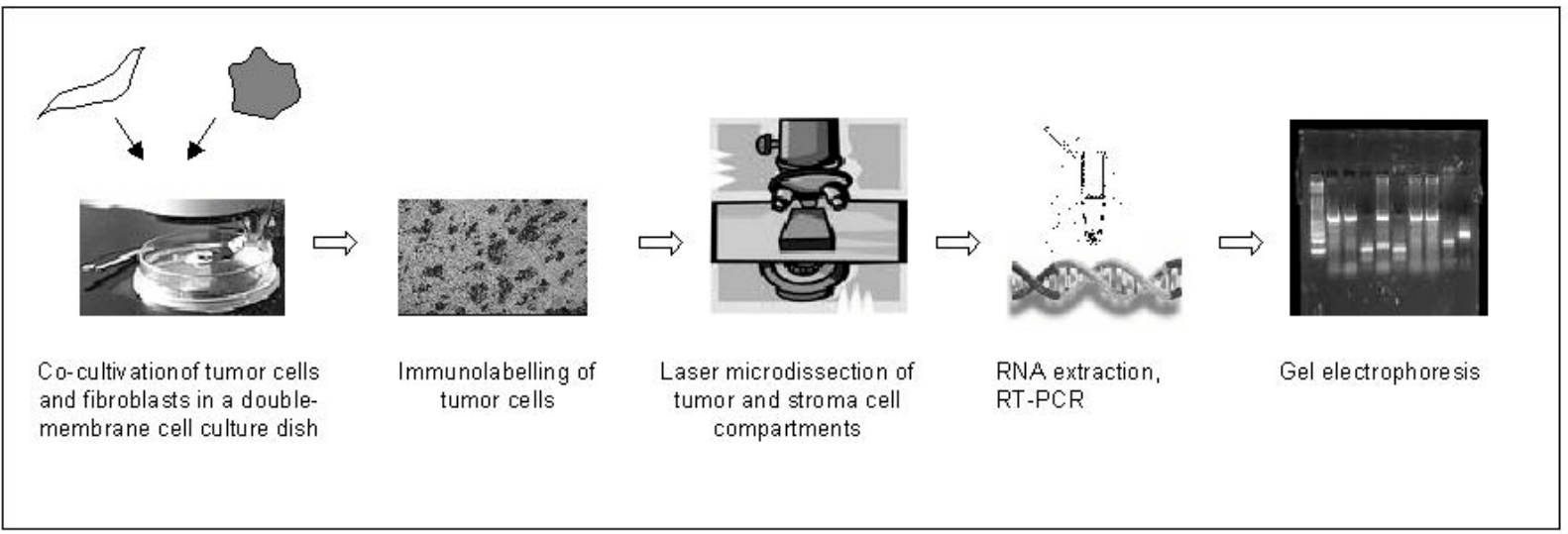

Fig. 1. Methodology flow chart. 
In our membrane-based co-culture model, fibroblasts were initially overlayed with tumor cells and co-cultivated for up to ten days. After this time immunolabeling revealed clearly separable cell compartments. We did not observe microscopically any cross-contaminating fibroblasts between the tumor cell layer and the membrane.

Laser capture microdissection: After identifying the tumor cells by immunostaining, about 5000 of each labeled and unlabeled (i.e., fibroblast) cells are laser microdissected.

Several systems for cell microdissection are available that mainly vary in the principle how to capture the dissected cells, in system configuration, and applications. Some systems offer the possibility of working with cells straight from a culture dish. Most allow to avoid touching the specimen by catapulting or dropping the laser dissected cells straight into a capture tube.

The instruments include an inverted microscope and either an infrared laser (PixCellßII from Arcturus, USA; Clonis ${ }^{\mathrm{TM}}$ from Bio-Rad, Calif., USA) or ultraviolet laser (PALM $®$ Microbeam from P.A.L.M. Bernried, Germany; Leica AS LMD from Leica Microsystems of Wetzlar, Germany; $\mu \mathrm{CUT}$ from MMI Heidelberg, Germany). Several different tissuecapture techniques are offered. The widely approached PixCell II from Arcturus employs a disposable cap, which is placed over the target tissue area on a slide. The caps are lined with thermoplastic film that forms a protrusion when hit by a laser pulse. The protrusion closes the gap between tissue and film, so that lifting the cap will remove the target cells, leaving them attached to the cap. MMIs $\mu$ CUT cuts around the tissue of interest, which is mounted on special membrane-covered slides and an adhesive layer in a collection cap to capture the sample is used during the collection process.

Capture using a special film is not the only way to collect an excised specimen. The P.A.L.M. MicroBeam ${ }^{\circledR}$ used in our experiments applies a technology called Laser Pressure Catapulting (LPC) where a 'photonic cloud' provides an energy pulse, which catapults the excised specimen into a microfuge tube cap in an entirely non-contact manner without encroachment of adjacent unwanted areas.

For any microdissection, an optimised staining and fixation protocol is needed that provides acceptable morphology and preserves the RNA integrity:

For subsequent RNA extraction, the microdissected cells should be captured in lysis buffer from the selected RNA isolation method or in RNA stabilisation solution (RNAlater ${ }^{\mathrm{TM}}$, Ambion Inc., USA). In RNAlater ${ }^{\mathrm{TM}}$, RNA is stable for 1 day at $37^{\circ} \mathrm{C}, 1$ week at $25^{\circ} \mathrm{C}, 1$ month at $4^{\circ} \mathrm{C}$ or indefinitely at $-20^{\circ} \mathrm{C}$ (as declared by the manufacturer).

Gene expression analysis: The RNA quality from the microdissected co-cultured cells is evaluated by RT-PCR for a housekeeping gene transcript and for the gene transcript used in immunostaining the tumor cell compartment (Dahse et al., 2002a).

Total-RNA extraction was performed using a protocol for limited amounts of starting material which combines a guanidinium thiocyanate based denaturation with a modified RNA precipitation and purification (Dahse et al., 2002b). Briefly, the cells were denaturated in denaturation solution from the Micro RNA Isolation Kit (Stratagene, La Jolla, CA, USA) and nucleic acids were isolated by ethanol after phenol/chloroform: isoamylethanol treatment. DNA is removed by RNase-free DNase enzyme in the presence of a Ribonuclease inhibitor (RNasin ${ }^{\circledR}$ Promega, Madison, WI, USA). The Total-RNA pellet is stored at $-70^{\circ} \mathrm{C}$.

Reverse transcription with $1 \mu \mathrm{l} 25 \mu \mathrm{M}$ Oligo $(\mathrm{dT})_{15}$ primer and cDNA amplification. is carried out with primers for the housekeeping gene transcript cyclophilin and for the laminin gamma 2 chain as a marker for epithelial tumor cells. For visualisation, $10 \mu \mathrm{l}$ amplification product is run on an ethidium bromide stained $2 \%$ agarose gel .

\section{RESULTS AND DISCUSSION}

The herein described techniques for co-culturing fibroblasts and carcinoma cells and separating them by the means of laser capture microdissection represent a novel basis to study tumor - stroma - interactions.

The membrane used for coculture does not influence cell growth. Differences in growth behaviour, cell morphology and immunostaining between cultivation on cell culture slides and cultivation on the membrane were not observed.

Theoretically there could be fibroblasts present under the cancer cells colonies in the coculture model, i.e., between the cancer cells and the membrane. This phenomenon has not been observed by microscopy in our experiments. Cancer cells are likely to displace the fibroblasts during the coculture process.

The immunolabeled tumor cells and fibroblasts could be easily distinguished and microdissected 
without cross - contamination (Fig. 2). A major advantage of the method can be seen in the feasibility to visualise cultured cells by normal microscopy and to follow the microdissection procedure in the microscope.

The amount of dissected cells depends on the cell type and the RNA transcripts of interest as starting material for downstream applications. Isolation of amplificable DNA from the microdissected cells can be performed with standard procedures. Analysis of RNA has been more troublesome and can be a major limiting step for downstream applications because of the loss of RNA quality and quantity during RNA sample preparation from a limited number of cells. A modified immunostaining procedure under RNAsefree conditions is essential in order to maintain RNA integrity.

As shown in our analysis of laminin gamma2 as an example for a tumor cell marker and of a housekeeping gene transcript, intact Total-RNA can be isolated with our protocol for limited amounts of starting material from a small number of co-cultivated cells that have been immunostained and laser microdissected.

Laminin cDNA was amplificable only in tumor cells and not in the co-cultivated fibroblasts indicating no cell-cross-contamination during microdissection.

Moreover, the immunohistochemical detection of the laminin gamma2 chain protein indicates not only the tumor cell compartment in our model. It embraces also the information that the gamma2 chain, a guide for epithelial invasion (Pyke et al., 1995) is expressed at the protein level.

Our method allows an easy approach to a more complex gene expression analysis potentially by cDNA microarray profiling. High-density arrays of oligonucleotides or cDNAs are among the most powerful tools for identifying tumor/stroma interactionassociated genes and dissecting regulatory patterns. Arrays work by hybridisation of labeled DNA or RNA to DNA molecules attached on a glass or plastic surface. Nucleic acid arrays (often called microarrays, gene-chip arrays or simply , chips') are generally produced by either robotic spotting of nucleic acids (PCR products, plasmids or oligonucleotides) onto a glass slide or in situ synthesis of oligonucleotides using photolithography. Due to technical achievements, arrays with more than 25000 different oligonucleotide probes or 10000 different cDNAs per square centimeter can now be produced.

Moreover, our method is not restricted to the investigation of tumor-stroma interactions. It represents a basic powerful technology that might be improved and extended for the DNA, RNA or protein level analysis of heterologous cell interactions also in other coculture models.
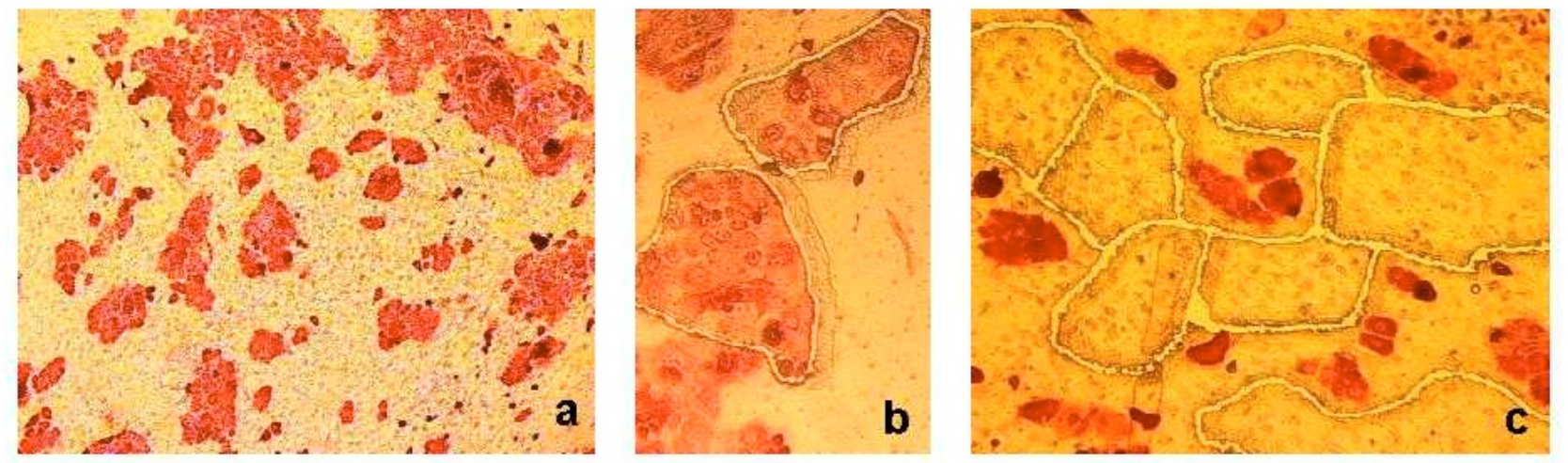

Fig. 2. Laser microdissection in the 2-D coculture model. Part (a) demonstrates a section of the membrane based 2-D coculture. Tumor cell areas are immunostained with the laminin 2 chain antibody GB3. Immunostained tumor cells (part b) or counterstained fibroblast areas (part c) are microdissected with the laser beam. The laser tracks are clearly visible and the precision of the procedure enables the dissection of the area of interest without cross contamination. 


\section{REFERENCES}

Dahse R, Berndt A, Haas KM, Hyckel P, Bohmer FD, Clauseen U, Kosmehl H. (2002a) Laser capture microdissection in 2-D co-culture models as a tool to study tumor-stroma interactions. Biotechniques. 33:474-75.

Dahse R, Berndt A, Kosmehl H (2002 b). Total-RNAbased target design for microarray analysis of defined tumor areas. Biotechniques 32:744-7.

Kunz-Schughart LA, Heyder P, Schroeder J, Knuechel R (2001). A heterologous 3-D coculture model of breast tumor cells and fibroblasts to study tumor-associated fibroblast differentiation. Exp Cell Res. 266(1):74-86.

Pyke C, Salo S, Ralfkiaer E, Romer J, Dano K, Tryggvason K (1995). Laminin-5 is a marker of invading cancer cells in some human carcinomas and is coexpressed with the receptor for urokinase plasminogen activator in budding cancer cells in colon adenocarcinomas. Cancer Res. 55(18):4132-9.

Stamenkovic I (2003). Extracellular matrix remodelling: the role of matrix metalloproteinases. J Pathol 200(4): 448-64. 